BMJ Surgery, Interventions, $\&$ Health Technologies

\title{
First in-human trial and prospective case series of an articulated laparoscopic camera system in minimally invasive surgery in gynecology: an IDEAL stage 1 and $2 a$ study
}

\author{
Tong Yow Ng (D) , ${ }^{1,2}$ Siew Fei Ngu, ${ }^{3}$ Tat Yan Deyoung Kam, ${ }^{1}$ Sai Yan Ng, ${ }^{4}$ \\ Ping Lai Benny Lo ${ }^{4}$
}

To cite: $\mathrm{Ng} \mathrm{TY}$, Ngu SF, Kam TYD, et al. First inhuman trial and prospective case series of an articulated laparoscopic camera system in minimally invasive surgery in gynecology: an IDEAL stage 1 and 2a study. BMJ Surg Interv Health Technologies 2022;4:e000117. doi:10.1136/ bmjsit-2021-000117

- Additional supplemental material is published online only. To view, please visit the journal online (http://dx.doi.org/10. 1136/bmjsit-2021-000117).

Received 24 September 2021 Accepted 15 February 2022

\section{Check for updates}

(c) Author(s) (or their employer(s)) 2022. Re-use permitted under CC BY-NC. No commercial re-use. See rights and permissions. Published by BMJ.

${ }^{1}$ Gleneagles Hospital Hong Kong, Hong Kong, Hong Kong

${ }^{2}$ The University of Hong Kong Department of Obstetrics and Gynaecology, Hong Kong, Hong Kong

${ }^{3}$ Department of Obstetrics and Gynaecology, The University of Hong Kong, Hong Kong, Hong Kong

${ }^{4}$ Precision Robotics (Hong Kong) Company Limited, Hong Kong, Hong Kong

Correspondence to

Dr Tong Yow Ng;

drtyng@mac.com

\section{ABSTRACT}

Objectives Precision Robotics' Sirius Robotic Flexible Endoscopic System is a new, fully integrated, compact three-dimensional laparoscopic camera system with a disposable single-use flexible tip that can change its viewing direction. This IDEAL Stage 1 and $2 a$ study assessed its safety, reliability and potential efficacy particularly for single incision laparoscopic surgery and vaginal natural orifice transluminal endoscopic surgery. Design Prospective single-institution, single-surgeon study.

Setting The study was conducted in a multispecialty hospital.

Participants Women aged 18-70 years scheduled for gynecological laparoscopic surgery were invited to participate. An information sheet and consent was available for the women and an informed consent was obtained. Thirteen participants completed this study. Interventions The laparoscopic procedures were done in the usual manner. The only difference was the Sirius System was used in place of the conventional laparoscope. All other procedures and instruments remained the same.

Main outcome measures Primary outcome was the proportion of women who successfully completed the intended procedure using the Sirius System without conversion to another camera system, camera users and surgeon's view and experience, and iterations and modifications to the system. Secondary outcomes were the incidence of intraoperative and postoperative complications during the first 6 weeks following surgery, and duration of surgery.

Results $85 \%$ (11/13) of women had their procedure completed successfully using the Sirius System. Two women required immediate conversion to the conventional laparoscope due to technical issues. There were no intraoperative complications. Users agreed that the improved field of view was beneficial for laparoscopic surgery. Iterative improvements were made in the imaging quality, user interface and manufacturing quality. Conclusions Sirius System has early indications for safety and efficacy for intermediate and major minimally invasive laparoscopic procedures in gynecology. Further

\section{Key messages}

What is already known about this subject?

- Minimally invasive surgery (MIS) in gynecology has progressed from multiport to single incision laparoscopic surgery (SILS) and vaginal natural orifice transluminal endoscopic surgery (vNOTEs).

- Conventional laparoscopic camera systems have a limited field of view.

\section{What are the new findings?}

- Sirius System, an articulated laparoscopic camera system shows early indications of safety and efficacy for MIS laparoscopic procedures in gynecology and can potentially replace a conventional laparoscope in the surgical workflow pending further studies.

\section{How might these results affect future} research or surgical practice?

- It is hoped that the Sirius System which has an articulated tip with $3 \mathrm{df}$, enabling C-shaped and Sshaped bending and providing a wider field of view compared with conventional laparoscopes may be particularly advantageous in minimally invasive SILS and vNOTEs procedures.

- The next step of clinical testing will be to conduct a prospective cohort IDEAL Stage $2 b$ (Exploration) trial involving multiple surgeons and multiple institutions.

studies are needed to confirm it can replace a conventional laparoscope in the surgical workflow.

Trial registration number NCT05048407.

\section{INTRODUCTION}

Minimally invasive surgery (MIS) is well established in gynecological surgery. Even for gynecological cancer surgery where overall survival must not be compromised, MIS is the gold standard for certain procedures. MIS laparoscopic total hysterectomy bilateral salpingoophorectomy is the preferred surgical management for early-stage carcinoma of 


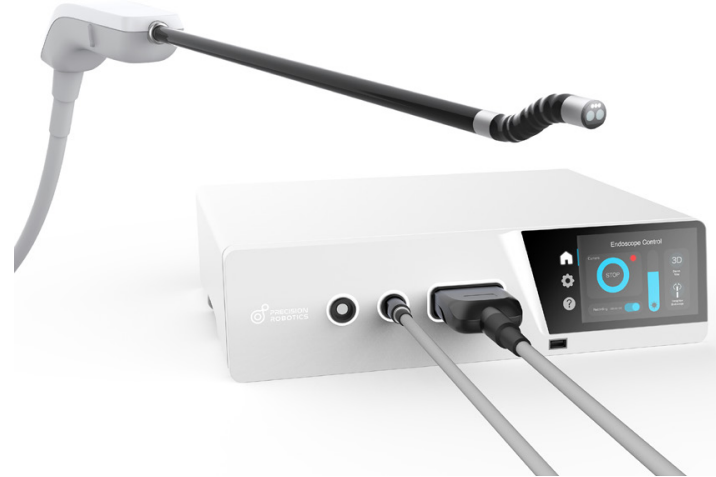

Figure 1 The Sirius Robotic Flexible Endoscopic System.

the corpus. ${ }^{1}$ Compared with laparotomy, MIS is associated with reduced morbidity and quicker recovery with no compromise to overall survival. ${ }^{2-5}$ With advancement in laparoscopic technology and techniques, MIS in gynecology has progressed from multiport to single incision laparoscopic surgery (SILS) and vaginal natural orifice transluminal endoscopic surgery (vNOTEs), thus further reducing morbidity and enhancing recovery. ${ }^{67}$

One of the challenges of MIS is the restriction of the range of surgical movement. This technical challenge is even more pronounced in the transition from multiport to SILS and vNOTEs where instrument crowding is a technical challenge. To overcome these technical challenges, articulated instruments are increasingly being used. ${ }^{8}$ Conventional laparoscopic camera systems have a limited field of view. Thus articulated camera systems such as the Olympus Endoeye Flex 3D (Olympus Medical Systems Corp) have come to market to address these issues. ${ }^{9}$

Precision Robotics' Sirius Robotic Flexible Endoscopic System is a new, fully integrated compact threedimensional (3D) laparoscopic camera system with a disposable single-use flexible tip that can change its viewing direction. ${ }^{10}$ The articulated tip has $3 \mathrm{df}$, enabling C-shaped and S-shaped bending, and providing a wider field of view compared with conventional laparoscopes (figure 1). This wider field of view is of particular advantage for SILS and vNOTEs. Presently available commercial systems do not have the same field of view or df. In addition to the disposable single-use robotic flexible laparoscope, the system is a fully integrated, compact system which consists of a video processor box with an integrated bright white light source, a handle and cable to connect the laparoscope to the video processor box, and a handheld joystick for control. ${ }^{11-14}$

From September 2019 to July 2020, three animal studies were performed by Precision Robotics in collaboration with National Taiwan University Hospital and Shanghai Ruijin Hospital. Using the Sirius System, expert surgeons successfully conducted the following procedures:

- Laparoscopic surgery-performed a cholecystectomy where the gallbladder was removed from an animal model laparoscopically.

- Trans-anal surgery-a small tissue sample was removed using an electronic knife to test the system's performance in a SILS operation with a porcine model.

- Orthopedics surgery-conducted a MIS-TLIF (minimally invasive surgery-transforaminal lumbar interbody fusion) operation on an animal cadaver.

Surgeons were able to complete their full surgical workflows using the system, and feedback was incorporated in refining the system's design.

In addition to animal studies, the system's safety was demonstrated through extensive testing by qualified testing centers in compliance with electromagnetic compatibility, safety and biocompatibility standards.

These preclinical studies have fulfilled the Pre-IDEAL stage of the IDEAL (Idea, Development, Exploration, Assessment, Long-term follow-up) framework. ${ }^{15-18}$ The aim of this IDEAL stage 1 and 2a first in-human trial and sequential prospective case series was to assess the safety, reliability and potential efficacy of a new articulated laparoscopic camera system-Precision Robotics' Sirius Robotic Flexible Endoscopic System in minimally invasive surgery in gynecology particularly for SILS and vNOTEs. The camera operators and surgeon's experience and view of the Sirius System were assessed. Iterative changes and modifications to improve the Sirius System were described. It was a sequentially reported prospective case series in a single institution, involving a single advanced gynecological laparoscopic surgeon and one team of nurses. It would then form the basis for an IDEAL stage $2 \mathrm{~b}$ exploration trial involving multiple surgeons and institutions.

\section{METHODS \\ Design}

This sequentially reported prospective single-institution, single-surgeon study was conducted from 18 May 2021 to 27 July 2021 at Gleneagles Hospital Hong Kong. Institutional Review Board ethical approval was obtained on 17 May 2021. All consecutive patients listed for MIS gynecological surgery by the principal investigator/surgeon fulfilling the study criteria were invited to participate in the study. The trial is registered at ClinicalTrials.gov.

The following baseline patient characteristics were recorded: age, diagnosis, name of surgery and date of surgery. On discharge from hospital, the following data were collected: date of admission, date of discharge, duration of surgery, blood loss, the successful completion of the procedure with the Sirius System without conversion to another camera system, intraoperative and postoperative complications. These data were extracted from the hospital database by the principal investigator/surgeon, and entered into a laptop computer protected by a password accessible only to the principal investigator/ surgeon.

Each nurse assisting the procedure and operating the Sirius Endoscope completed a Usability Questionnaire of the Sirius Endoscope System with a 1-5 Likert scale for each question (1=strongly disagree; $2=$ disagree; 
$3=$ neither agree nor disagree; $4=$ agree; $5=$ strongly agree), and entered free-text comments (online supplemental material 1). Anonymized video data of the surgical procedure were captured for analysis and validation. The recording were only of the surgical field, and only during the surgical procedure to prevent capturing any personal identifiable details. The video was reviewed for assessing the stability and safety of the procedure, and whether there were any modifications or unexpected changes to the procedure due to the Sirius System. The surgeon's comments on the efficacy, usability and safety of the Sirius System compared with a conventional laparoscope were recorded. Modifications and improvements to the Sirius System by Precision Robotics, based on feedback from the camera operators and surgeon during the course of the study, were recorded.

\section{Participants}

All consecutive patients aged 18-70 years listed for gynecological laparoscopic surgery by the principal investigator/surgeon during the duration of the study were invited to participate. An information sheet and consent for the study was available for the patient, and an informed consent was taken by the surgeon. It was emphasized that participation in the study was voluntary, and if they did not consent to the study, it would not affect their subsequent treatment and management. The participants were aware that this was an evaluation of a new endoscope. The safety aspects of the study including the availability of a conventional endoscope for immediate conversion if necessary was emphasized.
Exclusion criteria were pregnancy and/or failure to provide written informed consent for the study.

\section{Setting}

The study was conducted in Gleneagles Hospital Hong Kong, a multispecialty hospital. Prior to the commencement of the clinical trial, a comprehensive training program for the operating theater nurses on the usage of the Sirius System was conducted by Precision Robotics, and the Gleneagles Hospital Hong Kong Nurse Educator. This involved didactic as well as video and hands-on simulator training from 23 February 2021 to 19 March 2021.

A competency assessment was held from 20 March 2021 to 12 April 2021. This involved an online knowledge assessment, followed by assessment using a competency checklist on the usage of the Sirius System, as well as a simulation assessment on the simulator. In total 63 nurses were trained, and assessed to be competent before commencement of the clinical trial (online supplemental material 2).

The nine nurse assistants involved in this initial study had an average of 10.5 years (range: 5-15 years) of experience in assisting in laparoscopic surgeries. They had all completed the comprehensive training program on the usage of the Sirius System conducted by Precision Robotics and the Gleneagles Hospital Hong Kong Nurse Educator. Subsequently, the video recording of each procedure was reviewed, to ensure that the level of competence expected of the camera operators were achieved, and maintained throughout the study.

\section{Table 1 Patient demographics and procedures}

\begin{tabular}{|c|c|c|c|}
\hline $\begin{array}{l}\text { Case } \\
\text { no. }\end{array}$ & Diagnosis & Procedure & $\begin{array}{l}\text { Number of } \\
\text { abdominal ports }\end{array}$ \\
\hline 1. & Uterine fibroid & Total laparoscopic hysterectomy bilateral salpingo-ophorectomy (SILS) & 1 \\
\hline 3. & $\begin{array}{l}\text { Adenomyosis and ovarian } \\
\text { endometriosis }\end{array}$ & $\begin{array}{l}\text { Total laparoscopic hysterectomy left salpingo-ophorectomy, right } \\
\text { salpingectomy (multiport) }\end{array}$ & 3 \\
\hline 6. & Carcinoma of the corpus & vNOTEs hysterectomy bilateral salpingo-ophorectomy & 0 \\
\hline 7. & Carcinoma of the corpus & Total laparoscopic hysterectomy bilateral salpingo-ophorectomy (SILS) & 1 \\
\hline 8. & Ovarian dermoid cyst & Left ovarian cystectomy (SILS) & 1 \\
\hline 11. & Carcinoma of the corpus & $\begin{array}{l}\text { Total laparoscopic hysterectomy bilateral salpingo-ophorectomy and } \\
\text { bilateral pelvic lymphadenectomy (multiport) }\end{array}$ & 3 \\
\hline 12. & Uterine fibroid & Laparoscopic myomectomy (SILS) & 1 \\
\hline 13. & Ovarian cyst & Laparoscopic left ovarian cystectomy (SILS) & 1 \\
\hline
\end{tabular}

SILS, single incision laparoscopic surgery; vNOTEs, vaginal natural orifice transluminal endoscopic surgery. 
Table 2 Patient outcomes

\begin{tabular}{|c|c|c|c|c|c|c|}
\hline $\begin{array}{l}\text { Case } \\
\text { no. }\end{array}$ & Procedure & $\begin{array}{l}\text { Conversion to } \\
\text { conventional } \\
\text { laparoscope }(\mathrm{Y} / \mathrm{N})\end{array}$ & $\begin{array}{l}\text { Days in } \\
\text { hospital }\end{array}$ & $\begin{array}{l}\text { Duration of } \\
\text { surgery (min) }\end{array}$ & Blood loss (mL) & $\begin{array}{l}\text { Complications } \\
\text { within } 6 \text { weeks }\end{array}$ \\
\hline 1. & $\begin{array}{l}\text { Total laparoscopic hysterectomy bilateral } \\
\text { salpingo-ophorectomy (SILS) }\end{array}$ & $\mathrm{Y}$ & 5 & 78 & 50 & Nil \\
\hline 2. & $\begin{array}{l}\text { vNOTEs hysterectomy bilateral salpingo- } \\
\text { ophorectomy }\end{array}$ & $\mathrm{N}$ & 4 & 70 & 50 & Nil \\
\hline 4. & $\begin{array}{l}\text { Total laparoscopic hysterectomy bilateral } \\
\text { salpingo-ophorectomy (SILS) }\end{array}$ & $\mathrm{Y}$ & 3 & 148 & Insignificant & Nil \\
\hline 5. & Laparoscopic right ovarian cystectomy (SILS) & $\mathrm{N}$ & 2 & 43 & Insignificant & Nil \\
\hline 6. & $\begin{array}{l}\text { vNOTEs hysterectomy bilateral salpingo- } \\
\text { ophorectomy }\end{array}$ & $\mathrm{N}$ & 3 & 101 & 50 & $\begin{array}{l}\text { Vaginal wound } \\
\text { dehiscence }\end{array}$ \\
\hline 10. & $\begin{array}{l}\text { Laparoscopic bilateral ovarian cystectomy } \\
\text { (SILS) }\end{array}$ & $\mathrm{N}$ & 4 & 71 & Insignificant & Nil \\
\hline 11. & $\begin{array}{l}\text { Total laparoscopic hysterectomy bilateral } \\
\text { salpingo-ophorectomy and bilateral pelvic } \\
\text { lymphadenectomy (multiport) }\end{array}$ & $\mathrm{N}$ & 4 & 124 & Insignificant & Nil \\
\hline 12. & Laparoscopic myomectomy (SILS) & $\mathrm{N}$ & 3 & 90 & 50 & Nil \\
\hline 13. & Laparoscopic left ovarian cystectomy (SILS) & $\mathrm{N}$ & 3 & 40 & Insignificant & Nil \\
\hline
\end{tabular}

SILS, single incision laparoscopic surgery; vNOTEs, vaginal natural orifice transluminal endoscopic surgery.

The surgeon/principal investigator is an experienced, advanced gynecological laparoscopic surgeon, and gynecological oncologist with more than 20 years of clinical experience in laparoscopic surgery. He is a clinical advisor to Precision Robotics, and has been involved in the final development of the Sirius Endoscope from October 2020. He has been trained in the use of the Sirius Endoscope.

\section{Intervention, procedures and standard care}

The laparoscopic procedures were done in the usual manner. The only difference was for the study participants, the Sirius System was used in place of the usual conventional laparoscope. All other procedures and instruments including the number of ports remained the same.

For every procedure using the Sirius System, a conventional laparoscope was on immediate standby, to replace the Sirius System should there be an unanticipated equipment failure, so that the procedure could be completed without delay. Video recordings of each procedure were reviewed to ensure the safety of the Sirius System, and that there were no safety concerns arising from the use of the Sirius System.

\section{Outcome measures}

Primary outcome was measured by the proportion of women who successfully completed the intended procedure using the Sirius System without conversion to another camera system. The surgeon and nurses' views of the Sirius System was evaluated by the mean score of the Likert scale questionnaire and free-text comments. Modifications and changes to the Sirius System during the course of the study were recorded as part of the primary outcome measures.

Secondary outcomes were measured by the incidence of intraoperative complications, incidence of postoperative complications during the first 6 weeks following surgery and duration of surgery. Specifically, intraoperative complications directly related to the use of the Sirius System were documented. Duration of surgery was measured in minutes from urinary catheter insertion to closure of vaginal/abdominal wound. Intraoperative or postoperative complications were classified according to the Clavien-Dindo classification during the first 6 weeks of surgery. ${ }^{19}$

\section{RESULTS}

\section{Patient demographics and procedures}

A total of 13 consecutive patients were invited and consented to the study. No patient who was eligible was excluded, or declined to participate in the study. The median age was 44 years (range: $30-68$ years). Four 
Table 3 Usability Questionnaire of the Sirius Endoscope System using a 1-5 Likert scale for each question (1=strongly disagree; $2=$ disagree; $3=$ neither agree nor disagree; $4=$ agree; $5=$ strongly agree)

\begin{tabular}{|c|c|c|}
\hline Articulated tip & $\begin{array}{l}\text { Mean score } \\
\text { Likert scale }\end{array}$ & SD \\
\hline The presence of the $3 \mathrm{df}$ articulated tip is beneficial to the surgery & 3.93 & 0.47 \\
\hline When using the joystick to control the articulation, the response time is satisfactory & 3.43 & 0.76 \\
\hline The articulated tip movement including C-shaped or S-shaped bending can provide a wider field of view during surgery & 3.93 & 0.62 \\
\hline \multicolumn{3}{|l|}{ Imaging } \\
\hline Recognition of details/anatomy is satisfactory & 3.50 & 0.65 \\
\hline Illumination/brightness is sufficient & 3.29 & 0.83 \\
\hline Field of view is satisfactory & 3.93 & 0.47 \\
\hline Stability of the image is satisfactory (image glitch, disconnection...etc) & 3.29 & 0.73 \\
\hline Overall, the image quality is satisfactory & 3.36 & 0.50 \\
\hline \multicolumn{3}{|l|}{ Usability } \\
\hline The Sirius Endoscope System is compatible with the procedure & 3.71 & 0.73 \\
\hline The Sirius Endoscope System was easy to use & 3.43 & 0.85 \\
\hline When using the joystick to control the articulated tip, the response time is satisfactory & 3.07 & 0.62 \\
\hline Weight of the endoscope handle is satisfactory & 3.14 & 0.86 \\
\hline Ergonomics of the Sirius Endoscope is satisfactory & 3.29 & 0.61 \\
\hline Sterile and non-sterile areas are clearly identifiable during setup/surgery/takedown & 3.71 & 0.61 \\
\hline Overall I am satisfied with the Sirius Endoscope System & 3.57 & 0.65 \\
\hline
\end{tabular}

patients had carcinoma of the uterine corpus. The other nine patients had benign gynecological conditions: three patients had uterine fibroids, four patients had ovarian cysts, one patient had utero-vaginal prolapse and one patient had adenomyosis and endometriosis. Three patients had multiport laparoscopic procedures, eight patients had SILS and two patients had vNOTEs. Eight patients had a hysterectomy. Two patients in addition to hysterectomy had bilateral pelvic lymphadenectomy, as part of their staging procedure for carcinoma of the corpus. One patient had a SILS laparoscopic myomectomy for uterine fibroid. The remaining four patients had ovarian cystectomy. Patient and procedure details are in table 1 .

\section{Patient outcomes}

Of the 13 patients entered into the study, 11 (85\%) successfully completed their procedure using the Sirius System without conversion to a conventional laparoscope. The Sirius System was not used in Case 1 because during the preoperative checks, a labelling issue was identified in the case packaging for the single-use disposable laparoscope. The sterility of the instrument could not be verified, and therefore the case was not allowed to proceed using the Sirius System. An immediate conversion was done to the usual conventional laparoscope, and the procedure was completed without delay. The issue was resolved by providing additional documentation, and all subsequent batches of instruments from Case 5 onwards were provided with the correct labelling according to hospital standards.

For Case 4, the laparoscopic camera head was found to be defective during the procedure, causing a deterioration in image quality. An immediate conversion to the conventional laparoscope was done for safety reasons, and the procedure was completed without further delay. Investigations showed that the technical issue was due to a manufacturing defect. This was corrected, and the issue did not recur again in subsequent cases. The study was paused for 1 month while this issue was addressed by Precision Robotics.

There were no intraoperative complications. Blood loss was insignificant $(<5 \mathrm{~mL})$ in six patients $(46 \%)$ and $100 \mathrm{~mL}$ or less in the remaining seven patients. The median number of days in hospital was 4 days (range: 2-6 days). The median operating time was $75 \mathrm{~min}$ (range: 40-162 min).

No patient returned to the operating theater within 24 hours of surgery. At 6 weeks of follow-up, one patient $(8 \%)$ was re-admitted 14 days after the surgery for vaginal bleeding due to vaginal wound dehiscence requiring resuturing under general anaesthesia. This was classified as a Clavien-Dindo Grade IIIb complication. All patients remained well at 6 weeks after surgery. Details of patient outcome are in table 2. 
Table 4 Iterations and modifications to the Sirius System

\section{Case}

no.

\section{Procedure}

\section{Lessons learnt/improvement Notes}

1. Total laparoscopic hysterectomy $>$ Endoscope Inner packaging did not show the expiry date information. Resolved by bilateral salpingo-ophorectomy providing additional documentation, and all new batches were made to comply to (SILS) hospital standard.

$>$ During setup it was unsure that the connector was connected, until assisted by a Precision Robotics engineer. An improvement was made to the connector subsequently (figure 2).

2. vNOTEs hysterectomy bilateral >SIRIUS Endoscope was used with supporting documents to ensure sterility. salpingo-ophorectomy $>$ Though the image quality was sufficient to complete the surgery, the image was noted to be too red.

3. Total laparoscopic hysterectomy $>$ Scope insertion diameter tip was found to be slightly $>10 \mathrm{~mm}$ while trying to insert left salpingo-ophorectomy, right it into a reusable trocar, and it was found that the diameter was actually $10.6 \mathrm{~mm}$. As salpingectomy (multiport) such, the trocar was replaced with a $12 \mathrm{~mm}$ disposable trocar for the endoscope. $>$ Articulated bending was useful, but there was no indication on the screen of the position of the tip.

4. Total laparoscopic hysterectomy >Camerahead was found to be defective, and an investigation was initiated bilateral salpingo-ophorectomy subsequently. The root cause of the fault was found to be the incorrect connection (SILS) of the protective earth of the laparoscope head. All the camera heads from the same batch (008) were recalled. New batches were manufactured, and additional tests were carried out to ensure the same issues would not recur.

5. Laparoscopic right ovarian $>$ Endoscope label improvement with new endoscope production Batch (011). cystectomy (SILS) $>$ Software updates: Dynamic model/additional color mode (2 and 3)/connector connection label/single camera functional fix. (figures 2-4).

$>$ Grounding issues electrical hardware improvement/testing.

6. vNOTEs hysterectomy bilateral >Surgeon noted that after gaining more experience of the Sirius System, the salpingo-ophorectomy assistant nurse was able to use the articulated tip to follow and center the surgeon's operating field without moving the endoscope. This could facilitate VNOTEs and SILS procedures, where instrument clashes with conventional rigid endoscopes were one of the main hindrances.

$>$ Trial on the different color mode settings: Mode 1-normal (more realistic)/Mode 2-enhance sharpness (difference was noticeable)/Mode 3-hue shift to blue (color of the image looked unrealistic and therefore further improvement is required).

7. Total laparoscopic hysterectomy >Image quality was good and clear, until there was blood, when the image became bilateral salpingo-ophorectomy too red.

(SILS)

>Assistant nurse was not familiar with the use of the joystick and caused some issues stressing the importance of the learning curve. Overall, the articulated tip helped to avoid collision with other instruments.

8. Left ovarian cystectomy (SILS) No issues.

9. Total laparoscopic hysterectomy Image was too red and required setting the color mode to 3 to continue. bilateral salpingo-ophorectomy and bilateral pelvic lymphadenectomy (multiport)

10. Laparoscopic bilateral ovarian cystectomy (SILS)

Image glitch occurred when using the scope, and it required a change to another Sirius scope. The subsequent investigation found that this was due to the scope head inadvertent mechanical disconnection.

11. Total laparoscopic hysterectomy Endoscope batch implemented with true $10 \mathrm{~mm}$ diameter-production batch (012). bilateral salpingo-ophorectomy and bilateral pelvic lymphadenectomy (multiport)

12. Laparoscopic myomectomy (SILS)

No issues. It was noted that the single-use disposable laparoscope ensured sterility, and no downtime for sterilization in between cases 8,9 , and cases 11,12 , which were conducted one after the other.

13. Laparoscopic left ovarian No issues.

cystectomy (SILS)

SILS, single incision laparoscopic surgery; vNOTEs, vaginal natural orifice transluminal endoscopic surgery. 
Please ensure Laparoscope Handle connector is inserted fully over the silver line during set up.

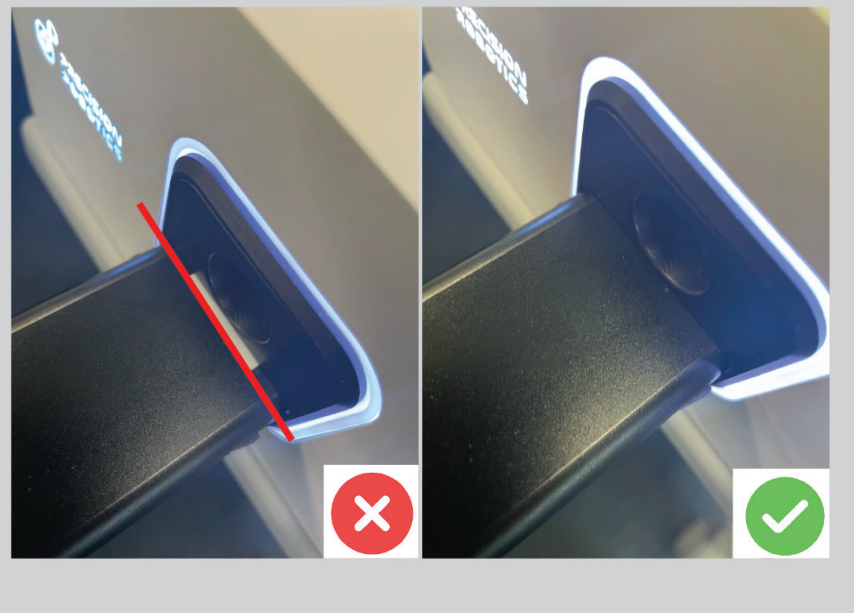

Figure 2 Label indicator to ensure connector is fully inserted into the video processor.

\section{Surgeon and camera operators' experience of the Sirius System}

Regarding the Usability Questionnaire of the Sirius Endoscope System, the individual questions and mean Likert scale score for each question is detailed in table 3. Comments on the individual cases, and suggestions for improvements were detailed in table 4 . The score was above 3 for all questions. The highest scores were for the articulated tip with its $3 \mathrm{df}$ of movement and improved field of view. It was the opinion that this was beneficial for the surgery. The surgeon noted in his comments for Case 6 (table 4) that this improved field of view, without moving the handle of the endoscope was particularly advantageous for SILS and vNOTEs procedures, where instrument clashing is a major technical hurdle for conventional endoscopes.

The lowest scores were for image quality, especially the color brilliance and color rendition. It was felt that the image color was too red compared with conventional endoscopes. Familiarity with the joystick control was also an issue, and improved with progress in the learning curve, and modifications to the graphical user interface from Case 5 onwards.

\section{Iterations and modifications to the Sirius System}

The iterations, and modifications to the Sirius System as the case series progressed, and feedback was obtained from the camera operators and surgeon, are detailed in table 4.

Case 1 showed a safety and setup issue, with inadequate labelling of the inner packaging, which did not show an expiry date of the scope. Therefore additional documents were needed to prove the sterility of the instrument, and these were verified by the hospital staff. This issue was resolved with correct labelling of the instruments from Case 5 onwards.

During setup for Case 1, it was also unclear if the connector was correctly connected, and it needed the assistance from a Precision Robotics engineer to verify the connection. This was resolved by incorporating a label indicator to provide a clear visual guide that the connector was correctly connected from Case 5 onwards (figure 2).

The articulated tip with its articulated bending, and wider field of view was helpful to the surgery. However it was noted that there was no indication on the surgical monitor of the position of the articulated tip. This was resolved with a software update and new graphical user interface, whereupon on successful connection of the laparoscope head, a simulated dynamic model would appear on the surgical monitor. This dynamic model provides real-time information on the current position of the articulated tip, and the joystick command status (figure 3).

Image quality and color rendition were issues raised by the surgeon, and he commented that the color of the image was too red. An image setting fix and software update were implemented from Case 5, which allowed the surgeon to choose the image settings and color modes (figure 4). In addition, Precision Robotics is performing detailed analysis on the color outputs of

Once the Laparoscope Head is successfully connected, a simulated dynamic model will appear on the surgical monitor. The dynamic model provides real time information about the current form of the Laparoscope as well as the Joystick command status.

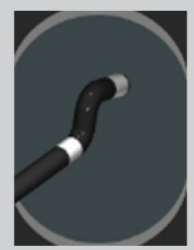

Idle

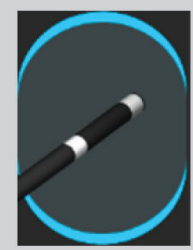

Straighten

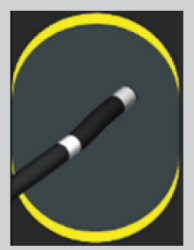

Moving

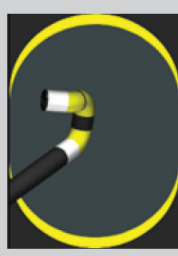

Max Angle

Reach

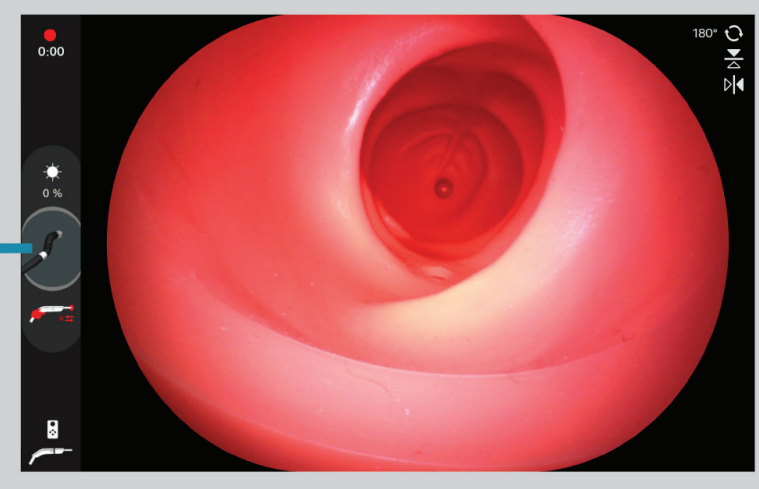

Figure 3 Dynamic model-included a three-dimensional model as part of the graphical user interface, providing real-time information of the articulated tip position for the user. 
The user can customize image settings and change color mode in settings page. E.g Image Flip, Swap Camera, Color Mode ...

Mode 1

Mode 3
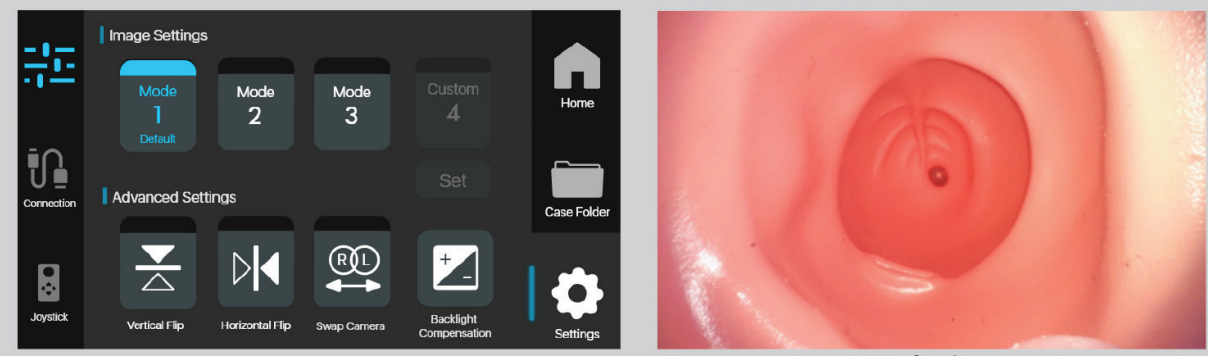

Default

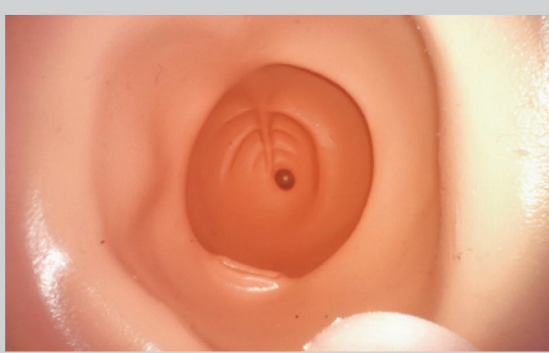

Enhanced Sharpness and Hue

Figure 4 Single camera fix - enable image setting - this is to provide additional image setting functions for the surgeon to choose to reduce the redness of the image and improve image quality.

the Sirius System, and of other conventional endoscopic systems, and the results will be used to refine the image processing algorithms.

Manufacturing quality, stability and robustness of the Sirius System was an issue shown in Case 3, 4 and 10. It was noted in Case 3 that while the stated diameter of the endoscope was $10 \mathrm{~mm}$, the actual diameter was measured to be $10.6 \mathrm{~mm}$. This quality control issue was addressed by the manufacturer in later batches of the instruments.

The camera head was found to be defective in Case 4 due to a grounding connection problem during the manufacturing process. All instruments in that batch were recalled, and an investigation was initiated by Precision Robotics. The study was suspended until the new batch of endoscopes were available. Based on the outcome of the investigation, new quality control, test procedures, and test plans were designed. Future endoscopes will need to undergo and pass these tests during the manufacturing process. The grounding connection design is also being reviewed and refined.

An image glitch was noted in Case 10, and subsequent investigations showed that it was due to inadvertent mechanical disconnection of the camera head. The buttons for releasing the camera head could be mistakenly pressed during handling of the endoscope. The mechanical design of the handle is being reviewed to minimize the risk of disconnection by incorporating a two-step lock. Further quality control methods and quality assurance procedures are being designed and will be imposed on the manufacturers of the Sirius System.

\section{DISCUSSION}

This is a first in-human, prospective sequential case series, IDEAL Stage 1 (Idea) and 2a (Development) study involving a single surgeon, in a single institution, with one team of nurses, of Precision Robotics' Sirius Robotic Flexible Endoscopic System which is a new, fully integrated, compact 3D laparoscopic camera system with a disposable single-use flexible tip that can change its viewing direction. The pre-clinical pre-IDEAL animal trial of the Sirius System, with feedback from surgeons demonstrated the safety of the system for human trial. A comprehensive pre-trial training program starting 3 months before the commencement of the trial was conducted for the operating theater nurses who would be handling the Sirius System, to ensure their competency and confidence in handling the Sirius System. The single surgeon involved in this case series is an experienced, advanced gynecological laparoscopic surgeon. He was involved in the final development of the Sirius System, and had been trained in its use. Video recordings of the procedures were reviewed to ensure that the competency of the team was preserved during the course of the trial, and there were no safety issues related to the use of the Sirius System.

This is an IDEAL Stage 1 and 2a proof of concept, and early development study. The successful completion of 11 of $13(85 \%)$ planned procedures in this sequentially reported, prospective case series, shows that the Sirius System can be used with indications of safety, and efficacy for intermediate and major MIS laparoscopic procedures in gynecology, and can potentially replace a conventional laparoscope in the surgical workflow. Technical issues which were corrected, were responsible for the conversion to conventional laparoscopes in two patients. The majority of the MIS procedures (10/13) were the more technically challenging SILS and vNOTES. There were no intraoperative complications. Blood loss was insignificant in $46 \%$ of patients $(6 / 13)$, and minimal $(\leqq 100 \mathrm{~mL})$ in the rest. There was no return to the operating theater within 24 hours in any patient. Most patients in this group including patients with cancer with staging procedures were discharged within 4 days, which is the norm for patients in an Asian setting, where patients elect to stay longer in hospital. ${ }^{20}$ Only one patient required re-admission for vaginal wound dehiscence, requiring re-suturing 14 days after surgery.

The Sirius System offers two distinct advantages compared with conventional laparoscopes in the surgical workflow. As noted by the surgeon in his comments on the cases (table 4), the single-use disposable laparoscope ensures sterility, and no downtime for sterilization in between cases. The articulated tip with $3 \mathrm{df}$, enabling C-shaped and S-shaped bending, and providing a wider field of view compared with conventional laparoscopes 
may be particularly advantageous in minimally invasive SILS, and vNOTEs procedures, where the clashing of instruments is a technical hurdle when using conventional endoscopes. The iterations, and modifications to the Sirius System during this IDEAL Stage 1 and 2a trial, enabled the Sirius System to reach stability for the last two cases. The improvements and modifications were in the areas of imaging quality and color, user interface, manufacturing process, and quality control and testing. There will be ongoing refinements, but no further major modifications are planned.

This IDEAL Stage 1 and 2a trial has shown that the Sirius System can be used with indications of safety, and efficacy in this early stage testing with a single surgeon, in a single institution, with one team of nurses, in a limited number of patients. Further assessment of the Sirius System, with multiple surgeons and nurses, in multiple institutions, with a larger number of patients more representative of the kind of variability expected of the patient population in the healthcare setting, will be needed in a prospective cohort IDEAL Stage 2b (Exploration) trial. There will also need to be more evaluation of learning curves, more outcome measures, reproducibility, and long-term follow-up before the Sirius System can replace conventional endoscopes.

Acknowledgements The management of Gleneagles Hospital Hong Kong for supporting the study, and providing the facilities for training of the operating theater nurses in the Sirius system. Rachel Wong, Assistant Director of Nursing, Operating Theater; Nurse Managers and Nurses of Operating Theater, Gleneagles Hospital Hong Kong for facilitating, and completing training in the Sirius System prior to study accrual. Engineers of Precision Robotics (Hong Kong) for maintenance and support of the Sirius System.

Contributors TYN is the main author, principal investigator/surgeon, design of study, editing of paper, analysis, data storage and processing. SFN participated in design of the study, edited paper, provided academic advice and guidance. TYDK participated in design of study, data analysis, designed and implemented training and assessment program for operating theater nurses for the Sirius System and edited the paper. SYN participated in design of the study, edited paper, data analysis, development and quality control of the Sirius System, participated in training and assessment of operating theater nurses and provided analysis of video images for improvement of the Sirius System. PLBL edited paper, participated in design of study and led development of the Sirius System.

Funding Precision Robotics (Hong Kong) Limited provided the Sirius System, supplied, and funded all the disposable single use articulated flexible endoscopes in the study. Precision Robotics was not involved in the recruitment, or management of patients.

Competing interests TYN is Clinical Advisor to Precision Robotics (Hong Kong) Limited, SYN is employee of Precision Robotics (Hong Kong) Limited, PLBL is employee of Precision Robotics (Hong Kong) Limited.

\section{Patient consent for publication Not applicable.}

Ethics approval This study involves human participants and was approved by HKU-GHK Institutional Review Board, Registration No: HKU-GHK IRB 2021-01. Participants gave informed consent to participate in the study before taking part.

Provenance and peer review Not commissioned; externally peer reviewed.

Data availability statement Data are available upon reasonable request. We would be delighted to share and publish our raw data.

Open access This is an open access article distributed in accordance with the Creative Commons Attribution Non Commercial (CC BY-NC 4.0) license, which permits others to distribute, remix, adapt, build upon this work non-commercially, and license their derivative works on different terms, provided the original work is properly cited, appropriate credit is given, any changes made indicated, and the use is non-commercial. See: http://creativecommons.org/licenses/by-nc/4.0/.

\section{ORCID iD}

Tong Yow Ng http://orcid.org/0000-0002-9424-5716

\section{REFERENCES}

1 Concin N, Matias-Guiu X, Vergote I, et al. ESGO/ESTRO/ESP guidelines for the management of patients with endometrial carcinoma. Int J Gynecol Cancer 2021;31:12-39.

2 Janda M, Gebski V, Brand A, et al. Quality of life after total laparoscopic hysterectomy versus total abdominal hysterectomy for stage I endometrial cancer (lacE): a randomised trial. Lancet Oncol 2010;11:772-80.

3 Obermair A, Janda M, Baker J, et al. Improved surgical safety after laparoscopic compared to open surgery for apparent early stage endometrial cancer: results from a randomised controlled trial. Eur $J$ Cancer 2012;48:1147-53.

4 Kondalsamy-Chennakesavan S, Janda M, Gebski V, et al. Risk factors to predict the incidence of surgical adverse events following open or laparoscopic surgery for apparent early stage endometrial cancer: results from a randomised controlled trial. Eur J Cancer 2012;48:2155-62.

5 Janda M, Gebski V, Davies LC, et al. Effect of total laparoscopic hysterectomy vs total abdominal hysterectomy on diseasefree survival among women with stage I endometrial cancer: a randomized clinical trial. JAMA 2017;317:1224-33.

6 Schmitt A, Crochet P, Knight S, et al. Single-Port laparoscopy vs conventional laparoscopy in benign adnexal diseases: a systematic review and meta-analysis. J Minim Invasive Gynecol 2017;24:1083-95.

7 Baekelandt J. Total vaginal notes hysterectomy: a new approach to hysterectomy. J Minim Invasive Gynecol 2015;22:1088-94.

8 Chern BSM, Lakhotia S, Khoo CK, et al. Single incision laparoscopic surgery in gynecology: evolution, current trends, and future perspectives. Gynecology and Minimally Invasive Therapy 2012;1:9-18.

9 Olympus-europa.com. ENDOEYE FLEX 3D LTF-190-10-3D Olympus Medical Systems, 2021. Available: https://www.olympus europa.com/medical/en/Products-and-Solutions/Products/Product/ LTF-190-10-3D.html [Accessed 5 Dec 2021].

10 Precision Robotics (HK) Ltd. Sirius laparoscope system, 2021. Available: https://surgicalrobot.com.hk/index.php?id=59 [Accessed 5 Dec 2021].

11 Berthet-Rayne P, Yang GZ. Vision based shape reconstruction of tendon driven snake-like surgical robots. 10th Hamlyn Symposium on Medical Robotics, 2017.

12 Berthet-Rayne P, Gras G, Leibrandt K, et al. The i² Snake Robotic Platform for Endoscopic Surgery. Ann Biomed Eng 2018;46:1663-75.

13 Shang J. An articulated universal joint based flexible access robot for minimally invasive surgery. 2011 IEEE International Conference on Robotics and Automation, Shanghai, 2011:1147-52.

14 Shang J, Payne CJ, Clark J, et al. Design of a multitasking robotic platform with flexible arms and articulated head for minimally invasive surgery. Rep U S 2012;2012:1988-93.

15 McCulloch P, Altman DG, Campbell WB, et al. No surgical innovation without evaluation: the ideal recommendations. Lancet 2009;374:1105-12.

16 Pennell CP, Hirst AD, Campbell WB, et al. Practical guide to the idea, development and exploration stages of the ideal framework and recommendations. Br J Surg 2016;103:607-15.

17 Hirst A, Philippou Y, Blazeby J, et al. No surgical innovation without evaluation: evolution and further development of the ideal framework and recommendations. Ann Surg 2019;269:211-20.

18 Bilbro NA, Hirst A, Paez A, et al. The ideal reporting guidelines: a Delphi consensus statement stage specific recommendations for reporting the evaluation of surgical innovation. Ann Surg 2021;273:82-5.

19 Dindo D, Demartines N, Clavien P-A. Classification of surgical complications: a new proposal with evaluation in a cohort of 6336 patients and results of a survey. Ann Surg 2004;240:205-13.

20 Wang Y, Deng L, Tang S, et al. vNOTES hysterectomy with sentinel lymph node mapping for endometrial cancer: description of technique and perioperative outcomes. J Minim Invasive Gynecol 2021;28:1254-61. 